\title{
Rethinking Research Impact Assessment: A Multidimensional Approach
}

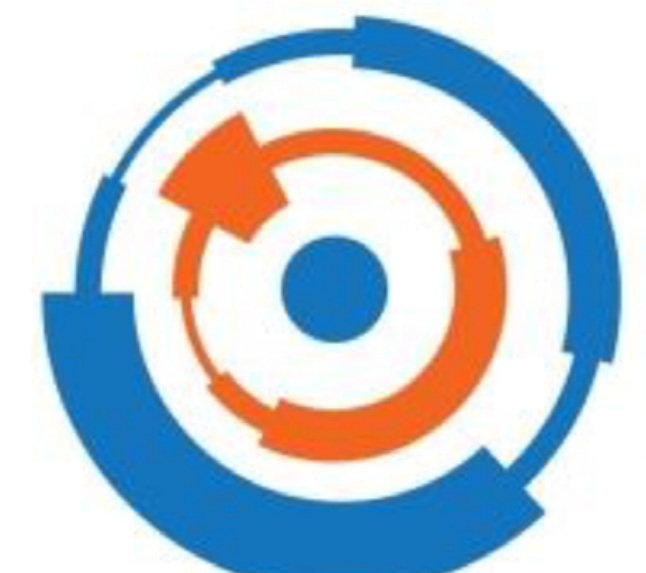
to impact from SSH research

Austrian EU Council Presidency Conference Impact of Social Sciences and Humanities for a European Research Agenda - Valuation of SSH in Mission-oriented Research

\section{Challenge}

An interest in the evaluation of research impact beyond academia has been observable worldwide. Several countries have introduced national research assessment systems which take into account this new element. However, these systems do not always offer a space to reflect on the ethical and reflexive sides of the impact generation and tend towards a 'one size fits all' model aimed at delivering measurable, quantifiable scores which can later be operationalized. We propose an ethical research impact self-assessment tool, as a contribution to the research impact agenda and with the aim of addressing the lack of models which stress reflexivity and ethics.

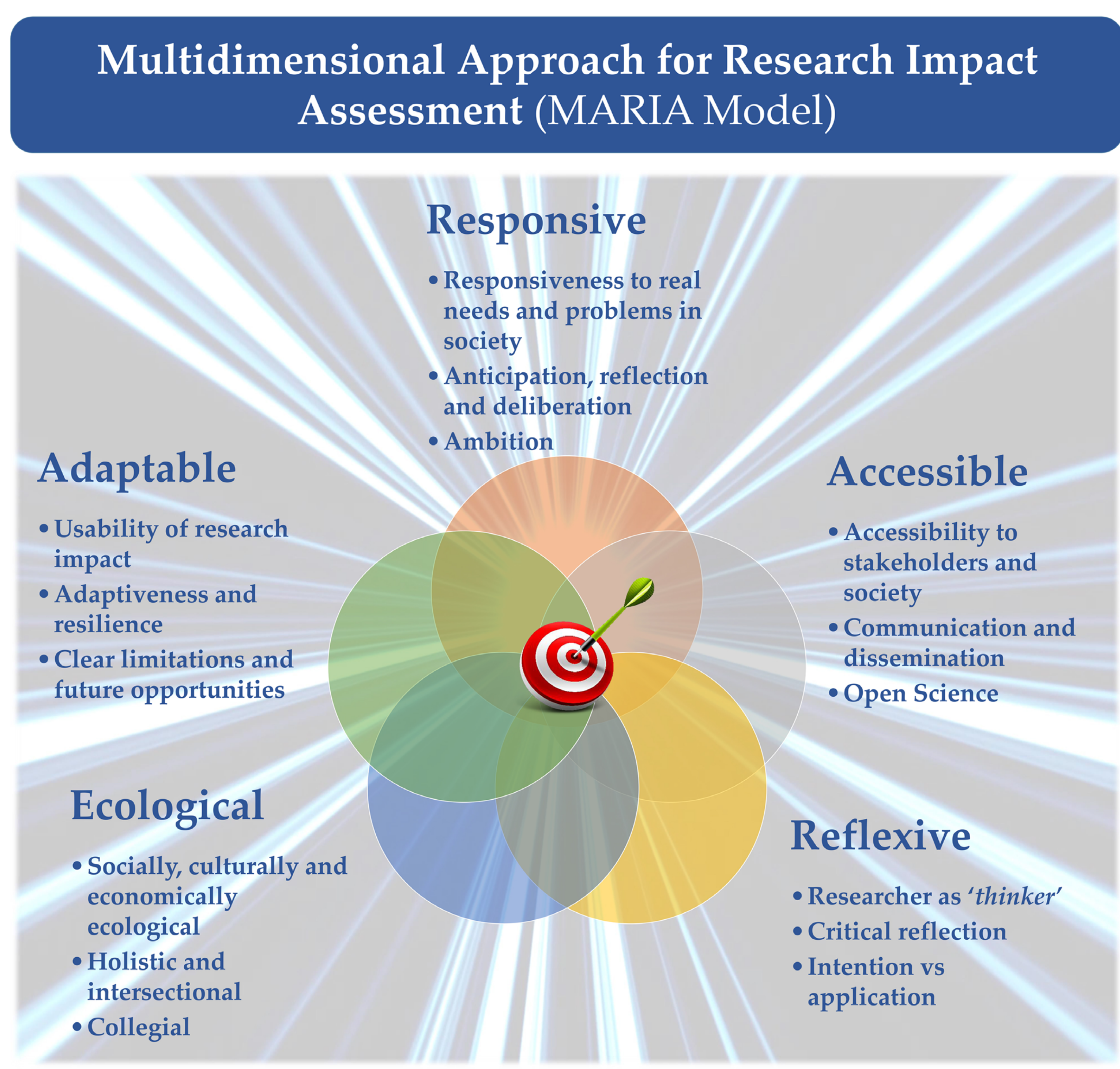

\section{Self-Assessment in Practice: MARIA Model in Use}

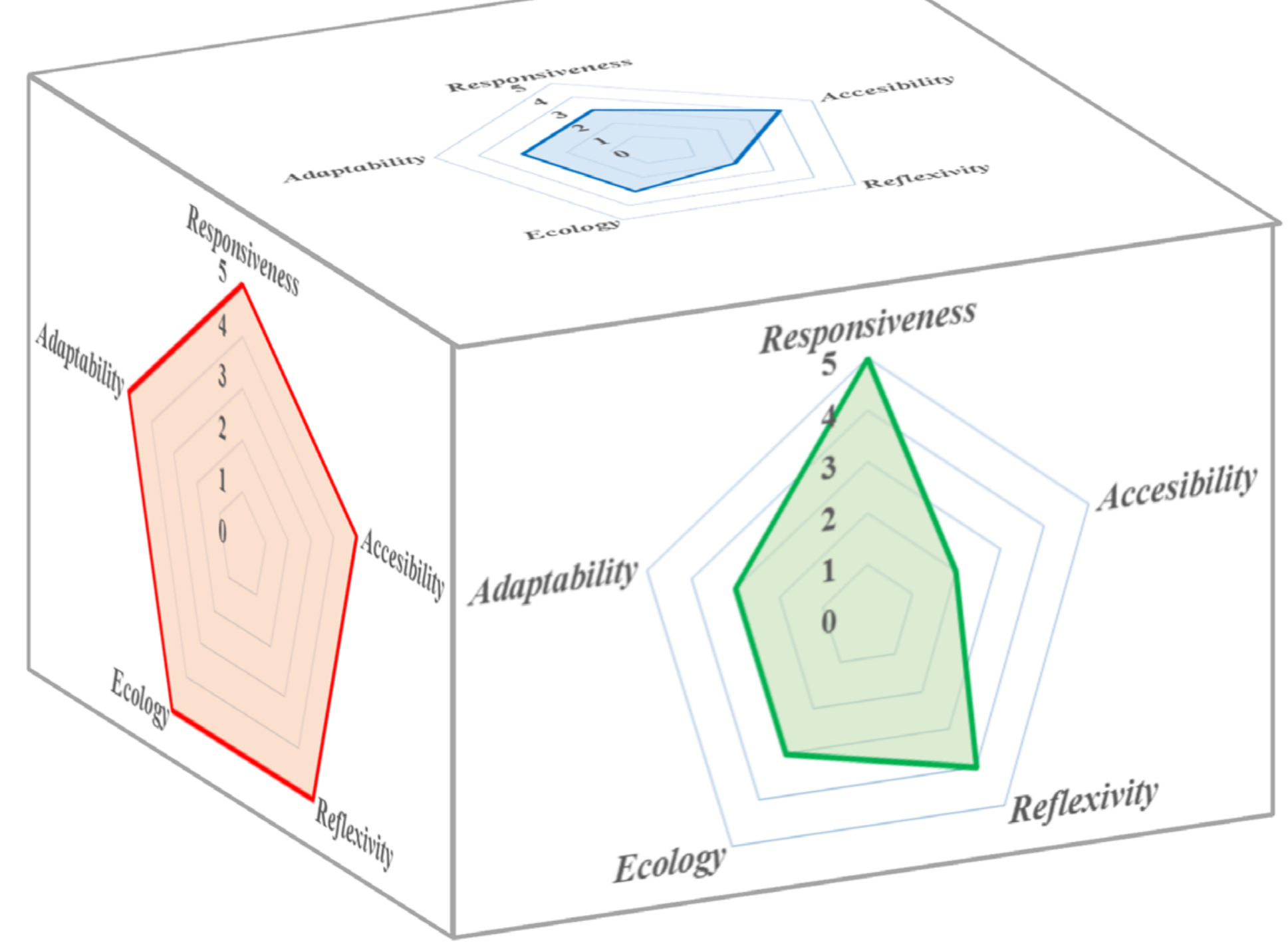

Examples of MARIA Pentagon

$\checkmark$ Does my research respond to real problems and needs in society? Am I contributing to current public debates?

$\checkmark$ Are my research outputs accessible to different stakeholders and society in general? Do I communicate and disseminate my research broadly and effectively?

$\checkmark$ Do I reflect on how comprehensive, well-planned and ethical my research is? Have I evaluated and critiqued my theories and analyses?

$\checkmark$ Does my research consider the relationships and connections among stakeholders and subjects? Was I collegial while conducting this research?

$\checkmark$ Is my research impact usable in different contexts and among different stakeholders? Am I aware of the limitations, future research opportunities and unanswered or emerging questions from my research?

\section{Conclusion}

What is lacking in the panorama of research evaluation is:

1) A framework to systematically reflect on the impact of one's own work (self-assessment).

2) A multidimensional model which recognizes the complexity of any impactful work.

3) A model which recognizes and reflects the ethical aspect of conducting impactful research.

The model we propose aims to address these gaps. Our proposal contributes to the ongoing learning process of research impact, in alignment with the context-based perspective of research assessment and in recognition of the need for a more holistic view in the observation and monitoring of interdisciplinary research. The article addresses pillars 1 (Valuation) and 3 (Assessing Impact) of the Austrian EU Council Presidency SSH-Impact Conference. 